\title{
Molecular Epidemiology of Giardia, Blastocystis and Cryptosporidium among Indigenous Children from the Colombian Amazon Basin
}

\author{
Angie Sánchez ${ }^{1,2}$, Marina Munoz ${ }^{2}$, Natalia Gómez ${ }^{1}$, Juan Tabares ${ }^{1}$, Laura Segura ${ }^{1}$, \\ Ángela Salazar ${ }^{1}$, Cristian Restrepo ${ }^{1}$, Miguel Ruíz ${ }^{1}$, Patricia Reyes ${ }^{1}$, Yuchen Qian ${ }^{3}$, \\ Lihua Xiao ${ }^{3}$, Myriam C. López ${ }^{1}$ and Juan D. Ramírez ${ }^{2 *}$
}

${ }^{1}$ Facultad de Medicina, Departamento de Salud Pública, Universidad Nacional de Colombia, Bogotá, Colombia, ${ }^{2}$ Grupo de Investigaciones Microbiológicas-UR, Programa de Biología, Facultad de Ciencias Naturales y Matemáticas, Universidad del Rosario, Bogotá, Colombia, ${ }^{3}$ Division of Foodborne, Waterborne, and Environmental Diseases, National Center for Emerging and Zoonotic Infectious Diseases, Centers for Disease Control and Prevention, Atlanta, GA, USA

\section{OPEN ACCESS}

Edited by:

Dongsheng Zhou,

Beiiing Institute of Microbiology and

Epidemiology, China

Reviewed by:

Anastasios D. Tsaousis,

University of Kent, UK

Antonio Frangipane di Regalbono,

University of Padua, Italy

*Correspondence:

Juan D. Ramírez

juand.ramirez@urosario.edu.co

Specialty section

This article was submitted to

Infectious Diseases,

a section of the journal

Frontiers in Microbiology

Received: 08 November 2016 Accepted: 06 February 2017

Published: 21 February 2017

Citation:

Sánchez A, Munoz M, Gómez N, Tabares J, Segura L, Salazar Á, Restrepo C, Ruíz M, Reyes P, Qian Y, Xiao L, López MC and Ramírez JD

(2017) Molecular Epidemiology of

Giardia, Blastocystis and

Cryptosporidium among Indigenous Children from the Colombian Amazon

Basin. Front. Microbiol. 8:248.

doi: 10.3389/fmicb.2017.00248
The incidence and prevalence of intestinal parasites in children is most likely due to lack of natural or acquired resistance and differences in behavior and habits closely related to environmental and socioeconomic determinants. The most important protozoa that parasitize humans are Giardia, Entamoeba, Blastocystis, and Cryptosporidium. These parasites present wide intraspecific genetic diversity and subsequently classified into assemblages and subtypes. The Amazon basin is the largest in the world and is the fifth freshwater reserve on the planet. Contradictorily, people living in these areas (Indigenous populations) have poor quality of life, which favors the infection of diseases of fecal-oral transmission. The aim of this work was to unravel the molecular epidemiology of Giardia, Blastocystis and Cryptosporidium across four communities (Puerto Nariño, San Juan del Soco, Villa Andrea and Nuevo Paraíso). We obtained 284 fecal samples from children under 15 years old that were analyzed by direct microscopy (261 samples) and Real Time PCR (qPCR) (284 samples). The positive samples for these protozoa were further characterized by several molecular markers to depict assemblages and subtypes. We observed a frequency of Giardia infection by microscopy of 23.7\% (62 samples) and by qPCR of $64.8 \%$ (184 samples); for Blastocystis by microscopy of $35.2 \%$ (92 samples) and by qPCR of $88.7 \%$ (252 samples) and for Cryptosporidium only $1.9 \%$ (5 samples) were positive by microscopy and qPCR 1.8\% (5 samples). Regarding the Giardia assemblages, using the glutamate dehydrogenase ( $g d h$ ) marker we observed Al, BIII and BIV assemblages and when using triose phosphate isomerase (tpi) we observed assemblages AI, All, BIII and BIV. In contrast, Blastocystis STs detected were 1, 2, 3, 4, and 6. Lastly, the species C. viatorum, C. hominis (with the subtypes IdA19 and laA12R8) and C. parvum (with the subtype IIcA5G3c) were identified. We observed a high profile of zoonotic transmission regarding the Giardia assemblages and Blastocystis STs/alleles. Also, we highlight the elevated frequency of infection by these two protozoans suggesting an active transmission in the area. Our findings reinforces the need to deploy better epidemiological surveillance systems for enteric pathogens in the area.

Keywords: Giardia, Blastocystis, Cryptosporidium, molecular epidemiology, assemblages, subtypes 


\section{INTRODUCTION}

Over 15 genera of protozoa parasitizing humans are known, some of these are considered natural commensal and others are related to intestinal infections responsible for generating symptoms in the infected hosts. The most frequent protozoans with cosmopolitan distribution are Giardia, Entamoeba, and Blastocystis. In Colombia, as reported in the latest national survey of intestinal parasitism in school population for the years 2012-2014, the most prevalent intestinal protozoan pathogen was Blastocystis with $57.7 \%$, the second was Entamoeba histolytica/dispar/moshkovskii complex (Entamoeba complex) with $17 \%$ and the third was Giardia with a national prevalence of 15.4\% (Ministerio de Salud y Protección Social, 2015). Additionally, Cryptosporidium has been reported with a frequency of $0.5 \%$ in the country. Currently, these are considered emerging opportunistic parasites of importance in public health at international level (Yoder et al., 2012). Factors associated with these parasitic infections are usually fecal contamination of soil and food, access to drinking water, wastewater use, lack of sanitation and vulnerable socio-economic conditions. Thus, maintaining the prevalence of intestinal parasitic infections in populations with these characteristics generating a latent risk to endure a dynamic transmission among its inhabitants (Ortiz et al., 2012).

One of the most common parasitic diseases in different geographical areas worldwide is giardiasis. It is estimated that 200 million people have symptoms and approximately 500,000 new cases per year are reported (Casero et al., 2015). In a retrospective study sought to describe the incidence of giardiasis in Colombia, for the years 2009-2013 revealed a report of 15,851 cases, of these, $50.3 \%$ were women; $58.4 \%$ were under 10 years old and $14.8 \%$ were $10-19$ years old. Some survey data indicate that in industrialized countries, the prevalence ranges between 2 and $5 \%$ and the rate for developing countries ranges from 20 to 30\% (Rodríguez-Morales et al., 2016). This disease is caused by Giardia intestinalis, an enteric pathogen that parasitize humans, domestic animals and wildlife. Studies in Giardia isolates have allowed to characterize the genetic diversity of the parasite, based on molecular markers such as the small subunit rRNA, glutamate dehydrogenase $(g d h)$, triose phosphate isomerase (tpi) and $\beta$-giardin (Ryan and Caccio, 2013), allowing to identify eight assemblages where A and $\mathrm{B}$ are related to infections in humans and animals and further subdivided into sub-assemblages: AI, AII, BIII, and BIV with no strict associations based on clinical presentation (Mayrhofer et al., 1995; Thompson, 2000). Assemblages C and $\mathrm{D}$ infecting dogs, assemblage $\mathrm{E}$ infecting ruminants and pigs; Assemblages $F$ infecting cats; $G$ infecting rodents and assemblage $\mathrm{H}$ infecting seals and gulls (Monis et al., 2009). These parasitic subpopulations are of epidemiological significance as a potential zoonosis and wide distribution in domestic animals that generate different transmission routes (Ramírez et al., 2015). This re-emerging parasite is a protozoan that produces symptomatic conditions such as diarrhea, abdominal pain and malabsorption syndrome (Stensvold, 2013; Roberts et al., 2014).
Likewise, the blastocystosis is an intestinal colonization caused by a stramenopile known as Blastocystis which is often observed in fecal samples from humans and animals; with a worldwide distribution frequency reaching $100 \%$ in developing countries and exceeding 56\% in developed countries (Scanlan et al., 2014). Blastocystis pathogenicity remains controversial, some authors suggest that the presence of infection might cause diarrhea, flatulence, bloating, urticaria and irritable bowel syndrome (IBS) (Casero et al., 2015). However, recent microbiome studies suggest that Blastocystis colonization is usually associated with a healthy gut microbiota, rather than with gut dysbiosis generally observed in metabolic or infectious inflammatory diseases of the lower gastrointestinal tract (Audebert et al., 2016). Also, a metagenomics approach showed that individuals with intestinal microbiota dominated by Bacteroides were much less prone to having Blastocystis-positive stool than individuals with Ruminococcus and Prevotella-driven enterotypes showing that the presence of Blastocystis might be benefitial for the human health (Andersen et al., 2015). Blastocystis has been divided into subtypes (STs) based on 18S rDNA polymorphisms and so far, 17 STs have been identified. The STs related to infections in humans and animals are 1-9 and 12, and the STs 10,11, 13-17 have only been detected in animals (Scanlan et al., 2014). However, the pathogenicity of this organism is under strong debate, mainly due to a high rate of asymptomatic carriers, the differences in host susceptibility, intestinal microbiota and/or different pathogenic potential of different genetic STs (Stensvold et al., 2012; Ramírez et al., 2014). In the case of Colombia, the reported studies suggest the existance of STs 1, 2, 3, 4, 6, and 7 (Ramírez et al., 2016).

Lastly, Cryptosporidium is a cause of cryptosporidiosis. A diarrheal disease affecting especially in children under 5 years and patients with immune deficiencies (Kotloff et al., 2013). In the developed countries, diarrhea is the most common reason for missing work, while in the developing world, it is a leading cause of death. Internationally, the mortality rate is $5-10$ million deaths each year (Nemes, 2009). In this scenario, Cryptosporidium is a major cause of diarrheal disease, globally (Shirley et al., 2012). Unlike many common causes of infectious enteritis, control and treatment of this infection are still problematic. Different reports indicated that cryptosporidiosis causes more than 99,000 deaths and 8.3 million disabilities (adjusted by years of life) by 2010; Most of these deaths and disabilities were common in developing countries (Ghenghesh et al., 2016). The course of infection may be intra and extra intestinal, symptomatic and asymptomatic in some individuals causing secretory diarrhea and malabsorption syndrome (Davies and Chalmers, 2009).

Molecular tools have been used to discriminate and subtype Cryptosporidium species. One of the popular subtyping tools is the DNA sequence analysis of the $60 \mathrm{kDa}$ glycoprotein (gp60, also called gp40/15); Gp60 is found on the surface of the apical region of invasive phases of the parasite, and one of the dominant targets for neutralizing antibody responses in humans (Wanyiri et al., 2007). Currently, nearly 20 Cryptosporidium species and genotypes have been reported in humans, including C. hominis, C. parvum, C. meleagridis, C. felis, C. canis, C. cuniculus, C. ubiquitum, C. viatorum, C. muris, C. suis, C. fayeri, C. andersoni, C. bovis, C. scrofarum, C. tyzzeri, C. erinacei, and 
Cryptosporidium I genotypes from horse, skunk, and chipmunk (Xiao, 2010; Liu et al., 2014; Ryan and Hijjawi, 2015). Within each subtype family, subtypes differ from each other mostly in the number of trinucleotide repeats (TCA, TCG, or TCT microsatellite; Xiao, 2010).

The incidence and prevalence of intestinal parasites in children is most likely due to lack of natural or acquired resistance and differences in behavior and habits closely related to environmental and socioeconomic determinants. In Colombia, there is information of the most common intestinal parasites but still lacks information about parasitic subpopulations (genotypes, subtypes, or assemblages) circulating in the population. In the case of the Colombian Amazon, it is not known in indigenous communities the frequency of different intestinal parasites that may be present in these ethnic groups, which are at risk of infection because of living in inappropriate conditions, associated with fecal contamination of soil and food, inadequate drinking water, wastewater use, lack of sanitation and vulnerable socioeconomic conditions. Thus, the prevalence of intestinal parasitic infections in populations with these sociodemographic characteristics is maintained, creating a risk for transmission dynamics among its inhabitants and a public health problem in the country (Rinne et al., 2005). Therefore, the aim of this study was to perform molecular diagnosis and genotyping from stool samples positive for Giardia, Blastocystis, and Cryptosporidium in children under 15 years of four indigenous communities in the Colombian Amazon.

\section{MATERIALS AND METHODS}

\section{Study Population}

Convenience sampling was conducted due to the geographical proximity of the individuals (Amazonas, Colombia) and age group (under 15 years). Therefore, we randomly selected 284 stool samples. The age average of the population included was 7.3 years (SD: 4.2 years-range 1-15). Individuals included in the study belonged to the municipality of Puerto Nariño located in the department of Amazonas, in three rural settlements linked to the jurisdiction of the Association of Indigenous Authorities of Tikuna, Cocama, and Yagua. According to the sampled communities the following distribution in sample number was obtained for every rural settlement: Nuevo Paraíso 10.9\% $(n=$ 31), Villa Andrea 15.1\% ( $n=43)$, San Juan del Socó 28,2\% $(n=$ $80)$ and the municipal capital Puerto Nariño $45.8 \%(n=130)$, which are located around the river Loretoyacu which has its confluence with the Amazon River (Figure 1).

\section{Ethical Considerations}

This study is an investigation of minimal risk to participants, in which the ethical standards set by the Ministry of Health of Colombia, the Juvenile Code and the Declaration of Helsinki of 2013 were followed. The parents/guardians of the minors provided informed consent and assent in order to obtain the sample. This project has approval certificate from the ethics committee of the Universidad Nacional de Colombia under the number: 002-012-15 issued on February 12, 2015.

\section{Collection, Conservation, and DNA Extraction of the Samples}

The samples were stored and labeled in plastic containers, transported to the laboratory of Parasitology of the Universidad Nacional de Colombia for further processing. They were divided into two parts, one part of the sample was used for diagnosis by microscopy; The second part was fixed in proportion weightvolume (1:4 stool:alcohol $70 \%)$ and were stored at $-20^{\circ} \mathrm{C}$ for DNA extraction from $300 \mu \mathrm{L}$ of stool by using the kit NORGEN Stool DNA Isolation, following the manufacturer's instructions.

\section{Diagnosis by Microscopy and Real-Time PCR for Identification of Giardia, Blastocystis, and Cryptosporidium}

The conventional routine microscopic diagnosis was conducted across 261 of 284 samples collected by direct examination in saline solution and lugol for identifying protozoa, and Ziehl Neelsen for identifying Cryptosporidium oocysts. Real time PCR was performed in all the 284 samples collected for Giardia, Blastocystis and Cryptosporidium by TaqMan system using previously reported primers and probes (Mejia et al., 2013). The qPCRs were performed in 96 wells MicroAmp (Applied Biosystems), reactions in a total volume of $9 \mu \mathrm{L}$ containing $3.5 \mu \mathrm{L}$ of Taqman $^{\mathrm{TM}}$ Mastermix (Roche), $1.0 \mu \mathrm{L}$ of species-specific primers $(10 \mu \mathrm{M})$ and primers of the internal amplification control (IAC) $(10 \mu \mathrm{M})$, and $0.4 \mu \mathrm{L}$ Taqman probes $(5 \mu \mathrm{M})$ (Giardia, Blastocystis, Cryptosporidium and the IAC), $0.3 \mu \mathrm{L}$ the water and $2.0 \mu \mathrm{L}$ of DNA. The samples were processed by duplicate in an Applied Biosystems 7,500 Fast equipment using default parameters of 40 cycles. The qPCR results were considered negative if the cycle threshold values $(\mathrm{Ct})$ were $>38$ (Mejia et al., 2013). To corroborate the Ct value, we conducted experiments to establish the dynamic range of our assay using standards from 10,000 to $1 \mathrm{fg} / \mu \mathrm{L}$. For quantification, plasmids containing the target sequences were cloned into the pGEM-T Easy Vector System I (Promega, UK), according to the manufacturer's instructions, and transformed into XL1-Blue Escherichia coli (Agilent Technologies, UK). The transformed colonies containing the plasmids were extracted by using the QIAprep Spin Miniprep Kit (QIAGEN, Valencia, CA). The purified plasmid DNA was quantified by using a Nanodrop and diluted to have a concentration range of 10,000 to $1 \mathrm{fg} / \mu \mathrm{L}$. The dynamic range established that the limit of detection was the proposed by Mejia et al. (2013). We employed as positive controls DNA from Giardia, Blastocystis and Cryptosporidium; and as negative controls fecal negative samples of patients from nonendemic regions that were previously tested by microscopy and qPCR.

\section{Genotyping for Identifying Giardia Assemblages, Blastocystis STs and Alleles, and Cryptosporidium Species and Subtypes}

As inclusion criterion, the genotyping was conducted in those positive samples obtained by real-time PCR for Giardia, Blastocystis, and Cryptosporidium. Any positive 


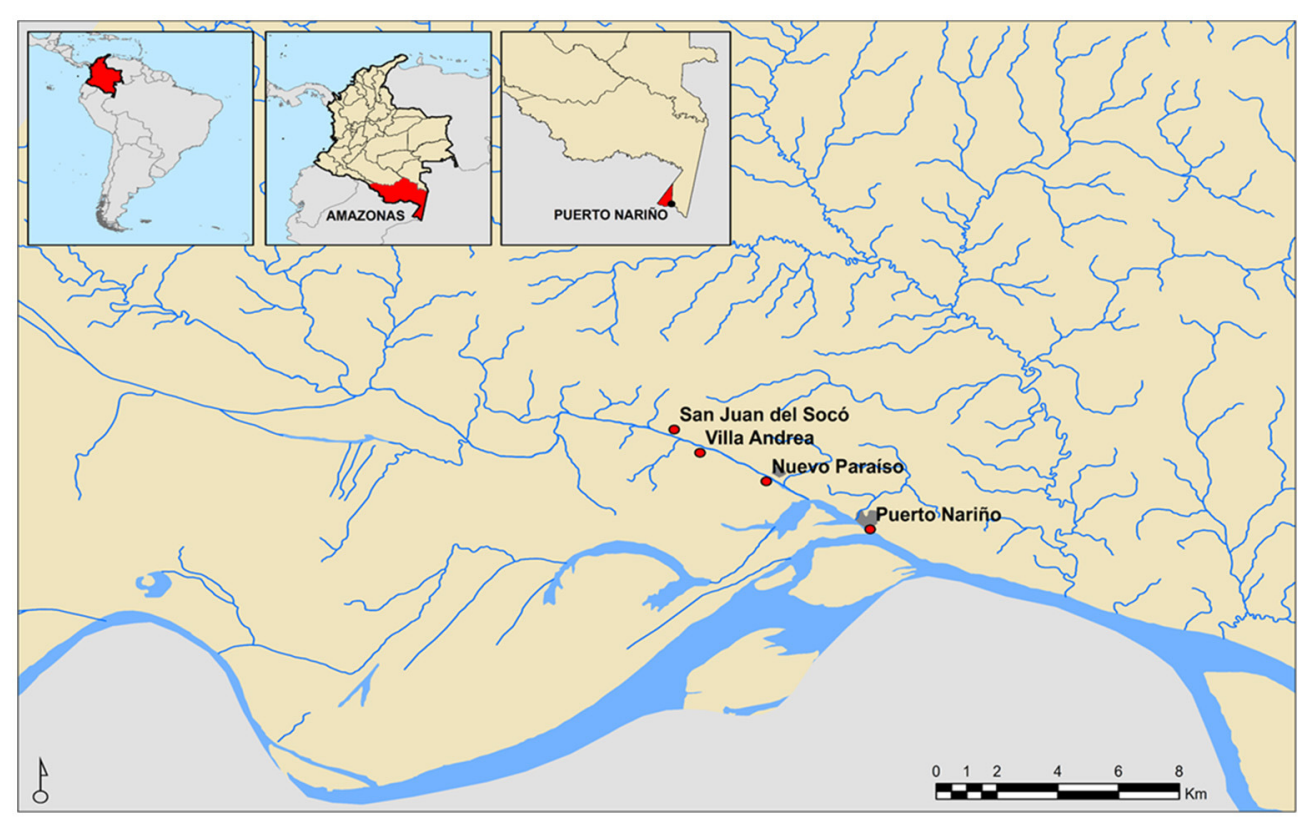

FIGURE 1 | Geographical location of Indigenous communities participating in the study.

sample was subjected to conventional PCR to obtain amplification products using primers of the following molecular markers: $g d h$ (Glutamate dehydrogenase) using primers GDHeF (5'-TCAACGTYAAYCGYGGYTTCCGT-3'), GDHiF (5'-CAGTACAACTCYGCTCTCGG-3') and GDHiR $\left(5^{\prime}\right.$-GTTRTCCTTGCACATCTCC- $\left.3^{\prime}\right)$ as reported elsewhere (Feng and Xiao, 2011) and tpi (triose phosphate Isomerase) using primers AL3543 (5'-AAATIATGCCTGCTCGTCG$\left.3^{\prime}\right), \quad$ AL3546 (5'-CAAACCTTITCCGCAAACC-3'), AL3544 (5'-CCCTTCATCGGIGGTAACTT-3'), AL3545 (5'GTGGCCACCACICCCGTGCC- $3^{\prime}$ ) as reported elsewhere (Feng and Xiao, 2011) for the identification of Giardia assemblages. In the case of Blastocystis, SSU rRNA amplification was performed with primers RD5 (5'-ATCTGGTTGATCCTGTCCAG-3') and BhRDr (5'-GAGTGCCTTTTT AACAACAAC G$3^{\prime}$ ) for identifying the STs and alleles of Blastocystis as previously recommended (Scicluna et al., 2006). In the case of Cryptosporidium, species were identified by using a small subunit rRNA-based PCR-restriction fragment length polymorphism genotyping tool (Chalmers and Katzer, 2013). Subtyping of C. hominis and C. parvum was based on sequence analysis of GP60 genes. Each specimen was analyzed by either method at least twice. Subtype families within C. hominis and $C$. parvum were determined on the basis of sequence differences in the nonrepeat region of the gene. Within each subtype family, subtypes differed from each other, mostly in the number of serine-coding trinucleotide repeats (TCA, TCG, or TCT microsatellite) located in the $5^{\prime}$ region of the gene. The previously established nomenclature system was used to differentiate subtypes within each subtype family (Xiao, 2010).

The PCR products were sequenced using the dideoxy-terminal method in a 310 Genetic Analyzer (Applied Biosystems) using both strands, the sequences obtained were edited and aligned in MEGA 5.0 (Tamura et al., 2011) and compared with reference sequences by BLAST. In addition, for Blastocystis, queries were performed on the database available for obtaining alleles and STs confirmation (http://pubmlst.org/blastocystis/). Phylogenetic reconstruction was performed using Maximum Likelihood with 1,000 bootstrap replicates with reference sequences contained in GenBank with the following accession numbers: AI (M84604), AII (AY178737), BIII (AF069059), BIV (AY178739), C (U60982), D (U60986), E (AY178741), E (AB182127), F (AB569384), G (AF069058), G (AY178745), H (GU176089) and rooted with G. microti (AY228649.1) and G. ardeae (AF069060). In the case of Cryptosporidium, sequences were compared with species and subtype control sequences harbored at CDC, Atlanta. The sequences were deposited under the accession numbers KX963577-KX963768.

\section{Genetic Diversity Indexes in Giardia and Blastocystis}

We calculated the genetic diversity indexes per community across the $g d h$ and tpi markers for Giardia and 18S rDNA marker for Blastocystis. $\pi$ and $\theta$ nucleotide diversity indexes and haplotype diversity were calculated in DNAsp v.5.0.

\section{Statistical Analysis}

Descriptive statistics was used to describe the main events of interest. Categorical variables are reported in terms of percentages, with corresponding Confidence Intervals (CI) at $95 \%$, calculated using the bootstrap method. In the case of continuous variables, means were calculated with their corresponding measures of dispersion (standard deviation-SD). The existence of association between categorical variables were 
evaluated using chi-square or Fisher, as appropriate tests. The distribution obtained by frequency for females was $49.2 \%$ ( $n$ $=128)$ and $50.8 \%(n=132)$ for male. The existence of association between the main results (epidemiological data and infection results) was evaluated using the categorical variables using chi-square or Fisher, tests, according to the characteristics of the data. In the case of the age of the individuals, was necessary to categorize the variable considering distribution percentiles obtaining four groups of 1-4 years, 5-8 years, from 9 to 12 years and one finally greater than or equal to 13 years old. First, the frequency of infection by age group, sex and community was obtained. Once identified the frequency and distribution of infection in each community were conducted bivariate analyzes to determine the existence of association between parasitic infections and sociodemographic factors (age, sex and community) for infected individuals. The associations between the results of infection (including the genetic diversity indexes (tpi, gdh, and 18S) within the groups of the same sex or age was identified per community. We employed chi-square or Fisher tests. In order to assess the presence and distribution of assemblages of Giardia and STs of Blastocystis, descriptive analysis were performed and association tests were developed according with the test previously mentioned, with the aim to compare against each demographic factor, likewise proceeded for STs present in Blastocystis. In the case of finding any degree of significance with STs, alleles belonging to it were faced with each variable in order to find some degree of association between a particular allele in populations. Lastly, we compared the presence of intestinal parasites such as Ascaris, Trichuris, hookworms and Entamoeba complex with Giardia assemblages, Blastocystis STs and Cryptosporidium species. The difference in the genetic diversity indexes was evaluated through comparison tests between independent samples, considering the results for each community. A value of $p<0.05$ was considered statistically significant for all hypothesis testing. Statistical analysis was performed using STATA version 10.1 (Stata Corp, College Station, TX).

\section{RESULTS}

\section{Frequency of Intestinal Parasites in Samples}

A total of 261 samples were analyzed by microscopy over the 284 collected because not enough sample was obtained for the direct analysis. In general, for stool samples collected in the school population of the four indigenous communities there were identified by microscopy other intestinal parasites such as: Whipworm in $62.0 \%(n=162)$, Ascaris lumbricoides in $46.3 \%$ ( $n$ $=121)$, hookworms in $22.2 \%(n=58)$ and Entamoeba complex in $20.7 \%(n=54)$. At the time of the analysis in order to find any kind of association between the intestinal parasites (Trichuris, Ascaris, hookworms and Entamoeba complex) and the Giardia assemblages, Blastocystis subtypes and Cryptosporidium species, no significant association was found between the frequencies of the other parasites reported by microscopy.

\section{Frequency of Giardia Infection}

Sixty-two samples were microscopy positive for Giardia $(23.7 \%$, CI:18.7-29.4) demonstrating that the highest percentage of infection was in the community of Villa Andrea (28.6\%) and Puerto Nariño (27.7\%) followed by San Juan del Socó (19.7\%) and Nuevo Paraíso (12.9\%). For Real-Time PCR, 184 samples (64.8\%, CI: 58.9-70.3) were positive, similarly observing the highest percentage of infection for Villa Andrea (74.4\%) and San Juan del Socó (65.0\%), followed by Puerto Nariño (62.3\%) and Nuevo Paraíso (61.3\%). The most common assemblage was AI (61\%) (Table 1; Figure 2), more frequently present in the community of San Juan del Socó (40.4\%), the second most common assemblage was BIII (17.2\%) present more frequently in the community of Villa Andrea (34.4\%). There was no significant association between the frequency of infection and communities. Across the positive samples, 120 sequences were analyzed with $g d h$ finding the AI, BIII and BIV assemblages showing a significant association between communities (Table 2). The tpi marker showed the presence of the four assemblages AI, AII, BIII, and BIV for which the most frequent assemblage was BIII (59.6\%) (Table 2), more frequently present in the community of Villa Andrea (28,1\%) followed by AI (4.9\%) more frequently present in the community of Nuevo Paraíso (16.7\%). No significant association between assemblages and communities were found with the markers employed. Additionally, the degree of agreement between the two markers used for genotyping (gdh and tpi) based on a chi-square test showed no association between the two markers $(p=0.135)$.

\section{Associations between Sociodemographic Variables and Giardia Assemblages}

The distribution of assemblages with $g d h$ identified for the age groups evaluated showed a significant association and when evaluating the independent variables was found that only the assemblage AI had an association being more frequently detected in children 9-12 years old (34.4\%) (Table 2). No significant associations between diagnostic methods and communities were found but when comparing the distribution of assemblages against communities was found an association, and when comparing with independent variables, the significant association was confirmed $(p<0.05)$ between the BIII assemblage and the community of Villa Andrea with $34.4 \%$ positive rate (Table 2 ). In the case of tpi marker, no association between age groups, gender and community assemblages was observed.

\section{Frequency of Blastocystis Infection, STs, and Alleles}

A total of 92 samples (35.2\%, CI: 29.4-41.3) over 261 samples were positive for Blastocystis by microscopy, where the highest percentage was obtained in Puerto Nariño (40.2\%) followed by Nuevo Paraíso (38.7\%), Villa Andrea (38.1\%) and San Juan del Socó $(25.0 \%)$, for the above there were not significant associations between infection and the communities (Table 1). Real-time PCR showed 252 positive samples (88.7\%, CI: 84.5-92.2) on a total of 284 samples and more frequently in the community of Nuevo Paraíso (93.5\%), followed by Villa Andrea (90.7\%), 
TABLE 1 | Frequency of infection by microscopy and qPCR of Giardia, Blastocystis and Cryptosporidium; and Giardia assemblages, Blastocystis subtypes and Cryptosporidium species/genotypes across the sampled individuals.

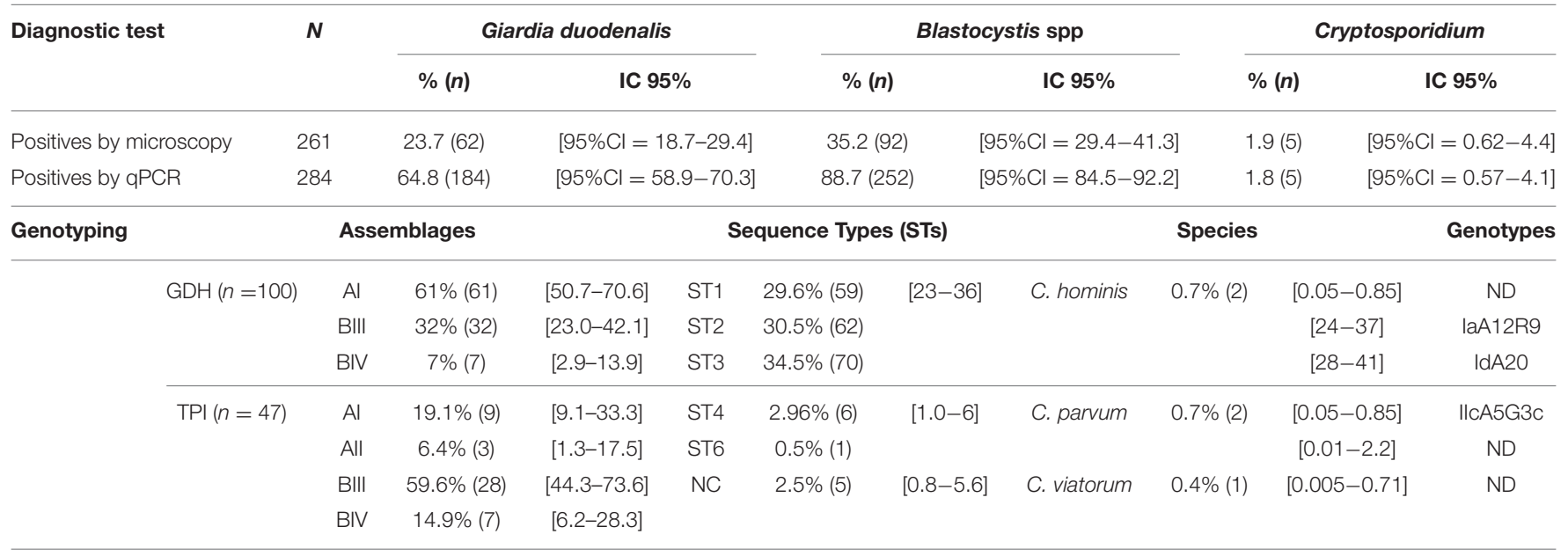

ND, Not determined.

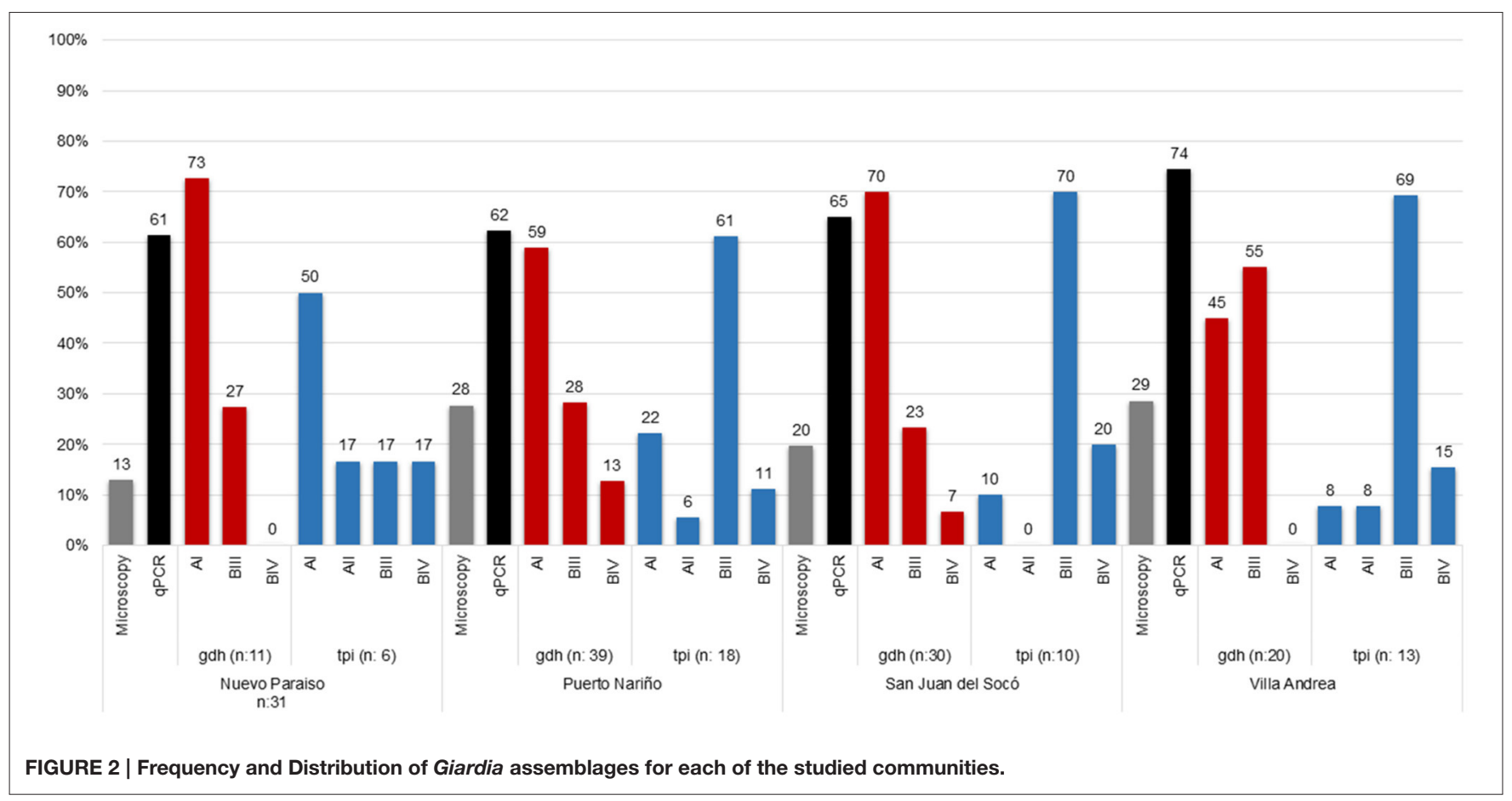

Puerto Nariño (87.8\%) and San Juan del Socó (87.5\%) (Figure 3). A total of 204 sequences were analyzed from the amplified products with the $18 \mathrm{~S}$ rRNA and we conducted $18 \mathrm{~S}$ barcoding observing ST3 (34.5\%), ST2 (30.5\%), and ST1 (29.6\%) (Table 1), with the highest frequencies across all the samples. These STs were more frequently present in San Juan del Socó (35.2 and 29.6\%) respectively. Additionally, ST 6 (0.9\%) was found in the community of Puerto Nariño and ST4 in Nuevo Paraíso (6.9\%), Puerto Nariño (2.6\%) and San Juan del Socó 1.4\%. Subsequently, we identified the alleles present in each ST, where allele 4 belonging to ST1 was the most common followed by allele 34 from ST3. Regarding the mixed infections, a total of $13.3 \%$ ( $n=$
27) were detected observing ST1 mixed with ST2, ST3, ST4, and ST5.

\section{Associations between Sociodemographic Variables, STs, and Blastocystis Alleles}

To evaluate the distribution of STs identified for the age groups, we found that ST1 was the only one which had an association with age again being reported more frequently in the group of children under 5 years (Table 2). Subsequently, when evaluating which one(s) of alleles of this ST is the one with more association with independent variables, we found that allele 4 is having a statistically significant association between the age group under 


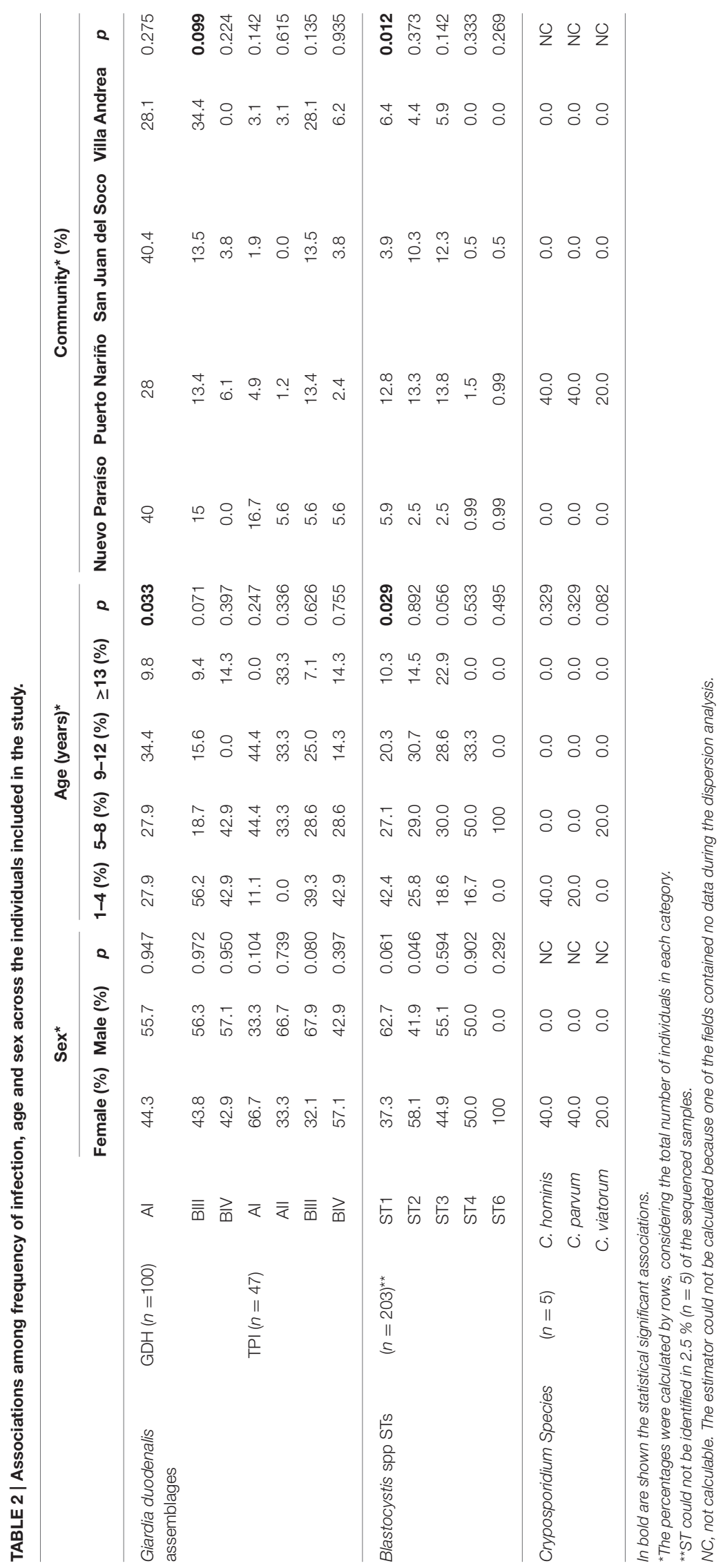




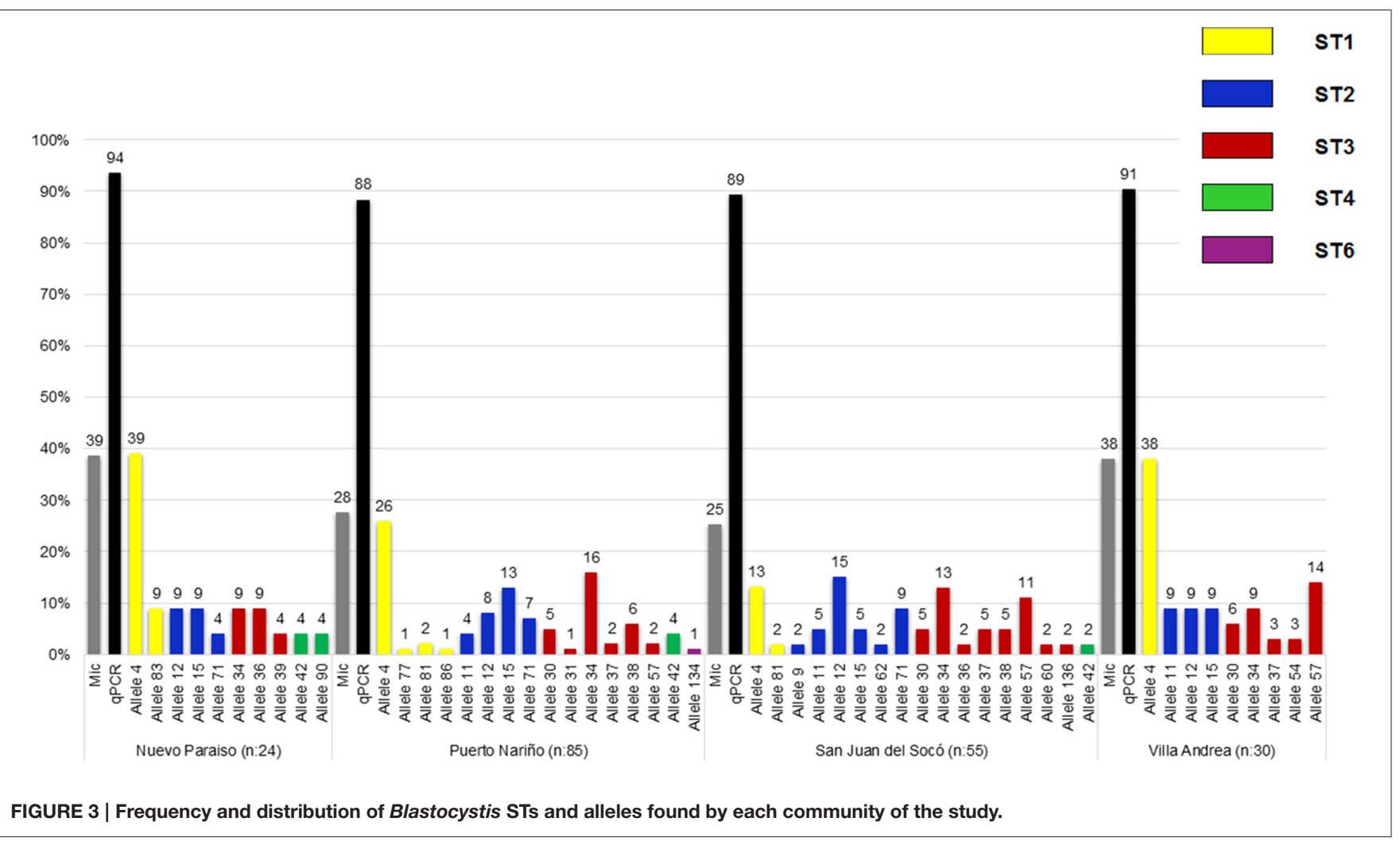

5 years. No significant association between the variables of sex and STs and distribution of alleles was observed. In conducting the analysis between the frequencies of the STs and communities, an association where the ST1 was the most frequent with $12.8 \%$ in the community of Puerto Nariño was found and to evaluate the variables independently it was found that ST2 and ST3 have a significant association being more common in the community of San Juan del Socó. When analyzing the variables independently, no significant association was found between them for either STs.

\section{Frequency of Cryptosporidium Infection, Species, and Subtypes}

A total of $1.9 \%(n=5)$ out of the 261 samples were positive for Cryptosporidium by microscopy. Real-time PCR showed 5 positive samples $(1.8 \%)$ on a total of 284 samples (Table 1), the samples positive by microscopy were the same positive by qPCR. The 5 samples were submitted to genotyping of the Gp60 for subtypes identification. The species C. viatorum $0.4 \%(n=1)$, C. hominis $0.7 \%(n=2)$ (with the subtypes IdA19 and IaA12R8) and C. parvum $0.7 \%(n=2)$ (with the subtype IIcA5G3c). All samples were detected in Puerto Nariño community. Additionally only the presence of Cryptosporidium in the female sex was found and no association was found with any of the sociodemographic variables. This is also attributable to the small number of positive samples obtained for this microorganism. On the other hand, when analyzing the association between positive samples for Giardia, Blastocystis and the other intestinal parasites detected by microscopy. We found no significant association with the presence of Cryptosporidium and the above mentioned parasites. Positive samples for C. hominis and C. parvum were found in the age groups of children under 5 years old and C. viatorum in children aged between 5 and 8 years (Table 2).

\section{Genetic Diversity Indexes in Giardia and Blastocystis}

We calculated the genetic diversity indexes per community across the $g d h$ and tpi markers for Giardia and 18S rRNA marker for Blastocystis (Table 3). Tests of significance between the results of community diversity indices (as independent samples), helped to identify a lack of statistically significant differences between the results of $\pi$ by community. In the case of $\theta$ and Hd profiles of differential association between the results obtained by the markers used for each species were found. The results of the index $\theta$ using the results of the marker gdh (G. intestinalis), obtained for the San Juan del Soco community, showed a significant difference to the results of Nuevo Paraiso and Villa Andrea. With the same score $(g d h)$ a significant difference between the results of San Juan del Soco and Puerto Nariño were found. The results of the second marker used for typing G. intestinal (tpi), showed a significant difference between the results obtained between San Juan del Soco, Puerto Nariño and Nuevo Paraiso. Significant differences were found with Hd for tpi between Nuevo Paraiso and the remaining three populations, but also between San Juan del Soco and Puerto Nariño. In Addition, Nuevo Paraiso showed significant differences with the results of the other three communities for both $\theta$ and $\mathrm{Hd}$, 


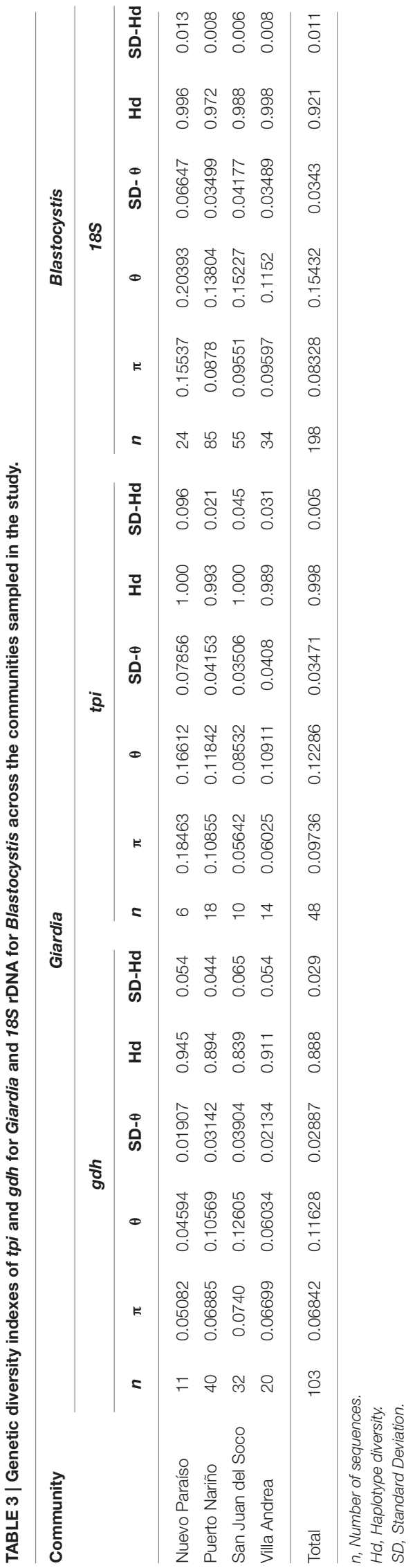

calculated for the sequences obtained for 18S (Blastocystis) and additional significant difference was identified for $\mathrm{Hd}$ calculated for San Juan del Soco and Puerto Nariño, from the results of 18 (Table 4). Despite these findings, no significant differences between the variances of rates among these populations were identified, so the hypothesis of equality of the results of the indices of genetic diversity at the population level is not rejected among these communities.

\section{Mixed Infections in Giardia and Blastocystis}

Mixed infections between G. intestinalis and Blastocystis were found by microscopy in $19.6 \%(n=29)$ being more common in Puerto Nariño with 27\% $(n=20)$, followed by Villa Andrea 16\% $(n=4)$, Nuevo Paraíso $14.3 \%(n=2)$ and San Juan del Socó 8.6\% $(n=3)$. At the time of the analysis against socio-demographic factors, community, sex and age were not statistically significant. In addition to the analysis for a possible association between subtypes of Blastocystis and G. intestinalis assemblages, did not show any association. The most frequent co-infections among STs of Blastocystis and G. intestinalis assemblages were evident; the STs 1, 2, and 3 present infection with assemblage AI, the ST3 was present in all assemblages, in the case of ST4 with AI, BIII, and BIV assemblages. Finally ST6 with a single report in Puerto Nariño presented BIII assemblage infection. A higher frequency of mixed infections in the community of Puerto Nariño and San Juan del Soco was observed (Figure 4).

\section{DISCUSSION}

The diagnosis of gastrointestinal parasites has traditionally been based on feces microscopy and the use of conventional techniques based on concentration for the subsequent visualization of parasitic stages. This leads to obtain a variability in sensitivity and diagnostic specificity, being totally dependent of the observer's expertise (Mejia et al., 2013). The object then is to improve and/or strengthen the diagnosis of parasitic infections, for which the use of molecular tools arises due to its high sensitivity allowing specific identification of various pathogens, i.e., species identification and subpopulations as is the case of Giardia assemblages, Blastocystis STs and Cryptosporidium species. This translates into specific and timely treatment to the organism and better clinical management of the patients. One of the applied/used strategies by different research groups is the molecular epidemiology. It has proven to be of great importance as it helps to understand the transmission dynamics and genetic determinants of parasitic infectious diseases (Ramírez et al., 2012). For example, recent reports have shown that giardiasis in childhood is associated with malnutrition, stunting and impaired cognitive development, regardless of the presence of diarrhea, prevalent among children two to five years old, according to several reports from developing countries (Montresor, 2002). It is clear that the incidence of intestinal parasites is higher in children than in adults because of the lack of natural resistance and differences in their behavior and habits (World Health Organization, 2012). The above closely related to the 


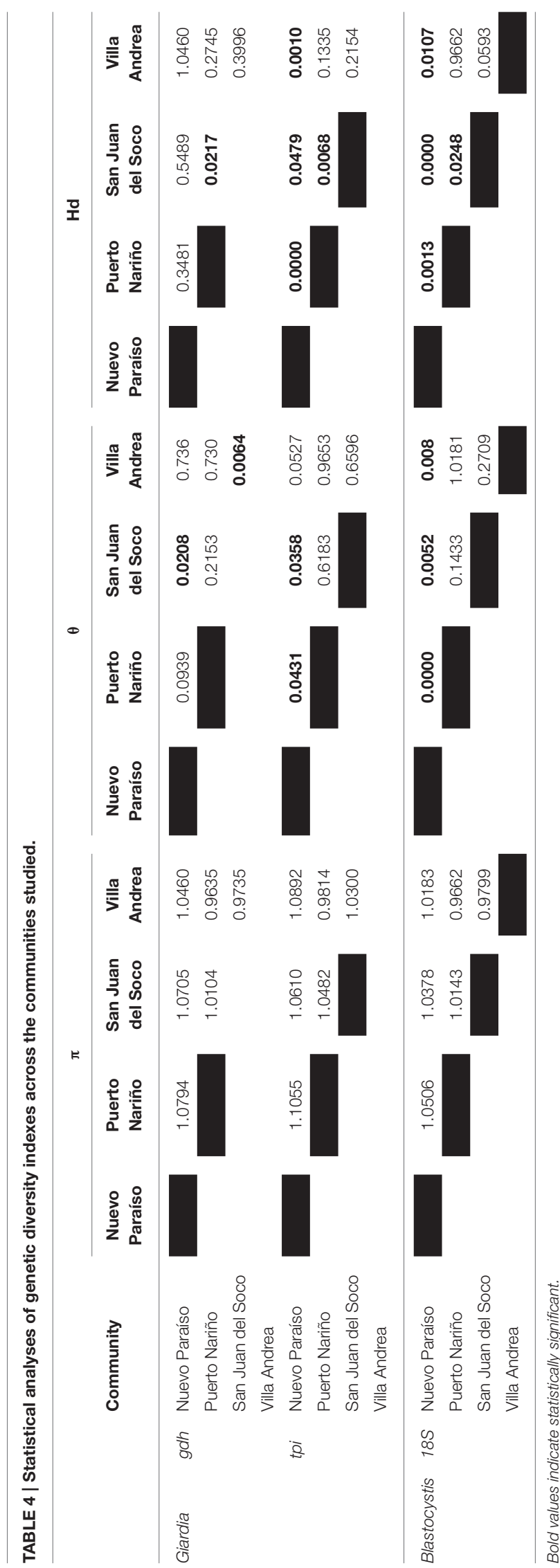

environmental determinants and socio-economic characteristics of a population, which generate a higher risk of infection. The World Health Organization (WHO) in its action plan raises early protection of children and vulnerable communities; detection, treatment and control of intestinal parasites are subject to follow, because this is one of the leading causes of morbidity and mortality in children worldwide (Haque et al., 2005; Ministerio de Salud y Protección Social, 2015).

An immune response, which can be individually variable and influenced by nutritional status, genetic factors, and repeated exposure to G. intestinalis suggesting that, contributes to low detection rates of Giardia seen in older children as well as an explanation to the asymptomatic infections (Forsell et al., 2016). Our results are in accordance with these premises, where we observed high rates of infection for Giardia (23.7\% by microscopy and $64.8 \%$ by qPCR) and Blastocystis (35.2\% by microscopy and $88.7 \%$ by qPCR) and also for other parasites such as Entamoeba, Ascaris, Trichuris, and hookworms. Also, the frequencies of infection for Giardia and Blastocystis are in accordance to other reports in Colombia showing that in the Amazon region Giardia has a frequency of $37.3 \%$ and Blastocystis has a frequency of $40.7 \%$, comparable to the frequencies of the country being for Giardia $15.4 \%$ and Blastocystis $57.7 \%$ (Ministerio de Salud y Protección Social, 2015). The differences are striking in terms of the sensitivity of the techniques which are also supporting the fact of using molecular methods instead of conventional microscopy as has been clearly stated by different authors (Bertrand et al., 2005; Asher et al., 2012; Mejia et al., 2013; Boadi et al., 2014; Ramírez et al., 2014). These can be explained by the intermittent excretion of cysts in Giardia and also the expertise of the operator who implements microscopy.

The geographical distribution of the $G$. intestinalis assemblages is intriguing since there is a lack of geographical structuring across the globe (in the case of the human-infective assemblages $A$ and $B$ ). In central and south-America, there exist areas with differential distribution of assemblages. Some reports from Mexico, Brazil and Colombia identifies higher frequencies of assemblage A (Eligio-Garcia et al., 2008; Kohli et al., 2008) while in Nicaragua, Argentina and also Colombia some authors report the predominance of assemblage B (Lebbad et al., 2008; Minvielle et al., 2008; Arroyo-Salgado et al., 2014; Ramírez et al., 2015). This suggests that the distribution is not geographically associated and is more linked to the socioeco-epidemiological factors of the population studied. This is corroborated by our findings where the most frequent assemblage was AI (61\%), contrasting the different findings in three sympatric regions of the country. For example in the Caribbean and central region the most frequent assemblage was B (Arroyo-Salgado et al., 2014; Ramírez et al., 2015) but in the Andean region assemblage A was more frequent (Rodríguez et al., 2014). Intriguingly, our results showed that the most frequent sub-assemblage was AI (61\%) that has been more associated to animals than humans. This could be attributed to the lack of water treatment of the population studied and a close contact with wild animals as is frequent in the amazon region. The presence of AI could be also possible due to contamination of public water with raw sewage from animal and human sources (Volotão et al., 2007; Helmy et al., 2009). It 


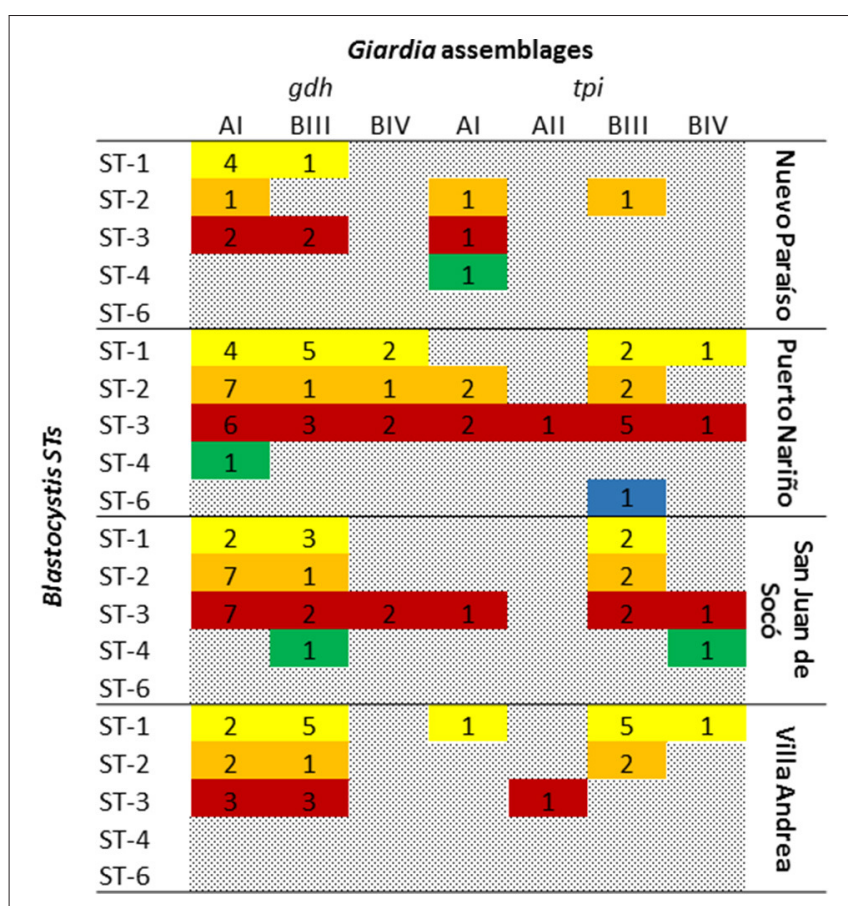

FIGURE 4 | Mixed infections between Blastocystis subtypes and G. intestinalis assemblages.

is clearly that the indigenous populations studied lack the access to potable water that is highlighted in our findings.

Our results also showed that after AI, assemblage BIII was the second most frequent. Curiously, this assemblage is highly associated to infections in dogs and cats which might be suggesting a strong zoonotic transmission in the community of Villa Andrea with $35.5 \%$ positivity in children under 5 years (Table 1; Lebbad et al., 2008; Ryan and Caccio, 2013). Regarding the genetic diversity indexes is observed that San Juan del Socó is the community with the highest genetic diversity and the second community with the highest rate of Giardia infection (65\%), and assemblage AI was the most frequent (40.4\%). Curiously, this community is the farthest from the municipality of Puerto Nariño suggesting that active enzootic transmission might be occurring representing a hot-spot of diversity where distinct genotypes from animals might be transmitted to humans.

The existence of many subtypes within families of $C$. hominis and C. parvum supports the complexity of Cryptosporidium transmission and its zoonotic potential in developing countries (Leav et al., 2002; Gatei et al., 2007). The subgenotypes considered anthroponotic (IIc and IIe) are the most common, along with the IId zoonotic subgenotype and the subgenotype IIc present in human infections (Akiyoshi et al., 2006; Sharma et al., 2013). These data supports our findings of IdA19 and IaA12R8 in C. hominis and IIcA5G3c in C. parvum. Herein, we show the first report of C. viatorum in Colombia; the data collected in different studies indicate the presence of C. viatorum in Asia (Nepal, Bangladesh, India, Pakistan, and potentially Dubai), Africa (Nigeria, Ethiopia and Kenya) and Central America and the Caribbean (Guatemala and Barbados) (Stensvold et al., 2015).
C. viatorum is currently the only species of Cryptosporidium that has been reported only in humans but the data are still insufficient to ensure that this species is restricted to human infections; also presumed presents a global distribution (Elwin et al., 2012). Previous studies indicate that the C. viatorum species has a genetic variation which may be related to differences in the geographical origin of the infection in different regions where it has been found (Elwin et al., 2012).

On the other hand, a high frequency of Blastocystis was observed in our study (88.7\%; Table 1), which agrees with previous reports of infection by Blastocystis in Colombia (Arias et al., 2010; Ramírez et al., 2014). In addition, this frequency is very similar to other countries in South America ranging from 40 to $70 \%$ in humans (Jiménez et al., 2012; Ramírez et al., 2016). Regarding the distribution of STs in humans, a study in South America showed a vast diversity occurrence of STs (1, $2,3,4,5,6,7,8$, and 12). In the case of Colombia have been reported STs 1, 2, 3, 4, 6, and 7 in humans (Malheiros et al., 2011; Ramírez et al., 2016). Our results show the predominance of STs 1,2, and 3 which is consistent with previous reports even in indigenous communities such as Mato Grosso, Brazil (Malheiros et al., 2011). Herein, we show several important findings which are the description of ST4 in indigenous communities since this ST is more common in Europe and less frequent in South America. In addition, ST6 that is more common in birds and may be an evidence of zoonotic transmission across the population studied (Stensvold et al., 2007). When the results of the $18 \mathrm{~S}$ alleles were retrieved, we observed that allele 4 from ST1 was frequent across the four communities; this allele has already been described in Colombia and with a frequency of $9 \%$ in all South America. Also, this allele has been reported in Didelphis marsupialis from Colombia supporting again a plausible zoonotic transmission (Ramírez et al., 2014, 2016). In the case of the ST2, alleles 12 and 15 were observed in all the communities as has already been described in South America with frequencies of 14 and $4 \%$ respectively, but intriguingly San Juan del Socó showed a higher number of ST2 alleles (6 in total) and in accordance with the genetic diversity indexes already described (Table 3; Figure 3). Also, allele 9 was found in this community and previously described in dogs and rats suggesting again a possible zoonotic profile (Ramírez et al., 2014). Regarding the ST3, the most frequent allele was 34, which has been reported in Colombia and with a frequency of $12 \%$ in South America (Ramírez et al., 2016). Interestingly, this allele has been associated to urticaria in Argentinean patients and also to cattle in Colombia (Ramírez et al., 2014; Casero et al., 2015). For ST4, we found alleles 42 and 90, the allele 42 has been described worldwide but allele 90; is novel in our country because dogs and cats have been found infected with alleles 42 and 133 (Alfellani et al., 2013; Stensvold and Clark, 2016). Finally, for ST6 we identified allele 134 in Puerto Nariño which is a novel description for the country because there is only a report of allele 122 (Ramírez et al., 2016).

According to the results obtained with mixed infections between Blastocystis and G. intestinalis (Figure 4) once again puts in evidence the need to employ techniques based on the use of different molecular markers to conduct a real identification of different subpopulations present in a specific area, and achieve 
associations regarding risk factors (Ryan and Caccio, 2013). Additionally, in the case of mixed infections, it is highlighted the frequency of infection with ST5, its presence in Latin America is only described in Bolivia 20\% $(n=8$; Ramírez et al., 2016), previously it had been reported in the UK, Australia and Pakistan (Yakoob et al., 2010; Wang et al., 2014). The importance of this ST is the zoonotic potential, as had previously been described in infections in pigs and cattle (Yan et al., 2007), reaffirming the importance in direct relation to human activities and possible infections that can occur between different epidemiological scenarios. In short, it is worth noting the fact of finding a high rate of mixed infections $13.7 \%$, this being an indicator of active transmission by generating new MLGs as observed in Trypanosoma cruzi (Ramírez et al., 2012).

The Amazon basin is the largest in the world and is the fifth freshwater reserve on the planet. Contradictorily, people living in these areas have poor quality of life, which favors the infection of diseases of fecal-oral transmission. Therefore, proper control of giardiasis, blastocystosis and cryptosporidiosis particularly in the Amazon region requires improving the quality of drinking water and reducing environmental contamination by feces, and control of the close contact with wildlife; despite of its high prevalence and its impact on health worldwide, enteric protozoa infections emerge as neglected because conditions are not taken into account as the controls used for infections caused by helminths. In different studies have shown that people living near a water body like a river, are at greater risk of becoming infected with some intestinal parasite as in the case of $G$. intestinalis and Blastocystis. In a recent study from the Brazilian amazon, the authors found a predominance of assemblage A which is in accordance with our findings (Coronato Nunes et al., 2016). This highlights the importance of our findings, and shows

\section{REFERENCES}

Akiyoshi, D. E., Tumwine, J. K., Bakeera-Kitaka, S., and Tzipori, S. (2006). Subtype analysis of Cryptosporidium isolates from children in Uganda. J. Parasitol. 92, 1097-1100. doi: 10.1645/GE-843R.1

Alfellani, M. A., Taner-Mulla, D., Jacob, A. S., Imeede, C. A., Yoshikawa, H., Stensvold, C. R., et al. (2013). Genetic diversity of Blastocystis in livestock and zoo animals. Protist 164, 497-509. doi: 10.1016/j.protis.2013. 05.003

Andersen, L. O. B., Bonde, I., Nielsen, H. B., and Stensvold, C. R. (2015). A retrospective metagenomics approach to studying Blastocystis. FEMS Microbiol. Ecol. 91:fiv072. doi: 10.1093/femsec/fiv072

Arias, J. A., Guzmán, G. E., Lora-Suárez, F. M., Torres, E., and Gómez, J. E. (2010). Prevalence of intestinal Protozoa in79 Children 2 to 5 years old from a state nursery in Circasia, Quindío. Infectio 14, 31-38. doi: 10.1016/S0123-9392(10)70090-4

Arroyo-Salgado, B., Buelvas-Montes, Y., Villalba-Vizcaino, V., and SalomonArzuza, O. (2014). [Genetic profiling of Giardia intestinalis by polimerase chain in human and dogs samples of Colombian Caribean Coast]. Enferm. Infecc. Microbiol. Clin. 32, 424-427. doi: 10.1016/j.eimc.2013. 07.016

Asher, A. J., Waldron, L. S., and Power, M. L. (2012). Evaluation of a PCR protocol for sensitive detection of Giardia intestinalis in human faeces. Parasitol. Res. 110, 853-858. doi: 10.1007/s00436-011-2565-3 the need to implement strategies for control and prevention in the communities studied because they are considered to be vulnerable indigenous communities.

In conclusion, we employed molecular methods and highresolution genotyping to unravel the transmission dynamics of Giardia, Blastocystis, and Cryptosporidium across four indigenous communities of the Colombian Amazon basin. We observed a high profile of zoonotic transmission regarding the Giardia assemblages and Blastocystis STs/alleles. Also, we highlight the elevated frequency of infection by these two protozoans suggesting an active transmission in the area. San Juan del Socó was the community with the highest genetic diversity for both pathogens and the closest to the jungle space. Our findings reinforces the need to deploy better epidemiological surveillance systems for enteric pathogens in the area and the use of molecular epidemiology for a vast comprehension of the transmission dynamics including the zoonotic potential of these species.

\section{AUTHOR CONTRIBUTIONS}

JR analyzed the data, revised the final version and supervised the project. NG, JT, LS, ASal, CR, and MR analyzed the samples by microscopy. ASan wrote the manuscript, analyzed the data and conducted the molecular biology experiments. MM conducted the statistical analyses. PR and ML revised the manuscript and analyzed the data. LX and YQ conducted the typing of Cryptosporidium.

\section{FUNDING}

We thank Dirección de Investigación Universidad Nacional de Colombia grant number 22946 for funding this project.
Audebert, C., Even, G., Cian, A., The Blastocystis Investigation Group, Loywick, A., Merlin, S., et al. (2016). Colonization with the enteric protozoa Blastocystis is associated with increased diversity of human gut bacterial microbiota. Sci. Rep. 6:25255. doi: 10.1038/srep25255

Bertrand, I., Albertini, L., and Schwartzbrod, J. (2005). Comparison of two target genes for detection and genotyping of Giardia lamblia in human feces by PCR and PCR-restriction fragment length polymorphism. J. Clin. Microbiol. 43, 5940-5944. doi: 10.1128/JCM.43.12.5940-5944.2005

Boadi, S., Polley, S. D., Kilburn, S., Mills, G. A., and Chiodini, P. L. (2014). A critical assessment of two real-time PCR assays targeting the (SSU) rRNA and gdh genes for the molecular identification of Giardia intestinalis in a clinical laboratory. J. Clin. Pathol. 67, 811-816. doi: 10.1136/jclinpath-2014-202224

Casero, R. D., Mongi, F., Sanchez, A., and Ramirez, J. D. (2015). Blastocystis and urticaria: examination of subtypes and morphotypes in an unusual clinical manifestation. Acta Trop. 148, 156-161. doi: 10.1016/j.actatropica.2015.05.004

Chalmers, R. M., and Katzer, F. (2013). Looking for Cryptosporidium: the application of advances in detection and diagnosis. Trends Parasitol. 29, 237-251. doi: 10.1016/j.pt.2013.03.001

Coronato Nunes, B., Pavan, M. G., Jaeger, L. H., Monteiro, K. J., Xavier, S. C., Monteiro, F. A., et al. (2016). Spatial and molecular epidemiology of Giardia intestinalis deep in the Amazon, Brazil. PLoS ONE 11:e0158805. doi: 10.1371/journal.pone.0158805

Davies, A. P., and Chalmers, R. M. (2009). Cryptosporidiosis. BMJ 339:b4168. doi: $10.1136 /$ bmj.b4168 
Eligio-Garcia, L., Cortes-Campos, A., Cota-Guajardo, S., Gaxiola, S., and Jimenez-Cardoso, E. (2008). Frequency of Giardia intestinalis assemblages isolated from dogs and humans in a community from Culiacan, Sinaloa, Mexico using beta-giardin restriction gene. Vet. Parasitol. 156, 205-209. doi: 10.1016/j.vetpar.2008.04.029

Elwin, K., Hadfield, S. J., Robinson, G., Crouch, N. D., and Chalmers, R. M. (2012). Cryptosporidium viatorum n. sp. (Apicomplexa: Cryptosporidiidae) among travellers returning to Great Britain from the Indian subcontinent, 2007-2011. Int. J. Parasitol. 42, 675-682. doi: 10.1016/j.ijpara.2012.04.016

Feng, Y., and Xiao, L. (2011). Zoonotic potential and molecular epidemiology of Giardia species and giardiasis. Clin. Microbiol. Rev. 24, 110-140. doi: 10.1128/CMR.00033-10

Forsell, J., Granlund, M., Samuelsson, L., Koskiniemi, S., Edebro, H., and Evengard, B. (2016). High occurrence of Blastocystis sp. subtypes 1-3 and Giardia intestinalis assemblage B among patients in Zanzibar, Tanzania. Parasit. Vectors 9:370. doi: 10.1186/s13071-016-1637-8

Gatei, W., Das, P., Dutta, P., Sen, A., Cama, V., Lal, A. A., et al. (2007). Multilocus sequence typing and genetic structure of Cryptosporidium hominis from children in Kolkata, India. Infect. Genet. Evol. 7, 197-205. doi: 10.1016/j.meegid.2006.08.006

Ghenghesh, K. S., Ghanghish, K., BenDarif, E. T., Shembesh, K., and Franka, E. (2016). Prevalence of Entamoeba histolytica, Giardia lamblia, and Cryptosporidium spp. in Libya: 2000-2015. Libyan J. Med. 11:32088. doi: 10.3402/ljm.v11.32088

Haque, R., Roy, S., Kabir, M., Stroup, S. E., Mondal, D., and Houpt, E. R. (2005). Giardia assemblage A infection and diarrhea in Bangladesh. J. Infect. Dis. 192, 2171-2173. doi: 10.1086/498169

Helmy, M. M., Abdel-Fattah, H. S., and Rashed, L. (2009). Real-time PCR/RFLP assay to detect Giardia intestinalis genotypes in human isolates with diarrhea in Egypt. J. Parasitol. 95, 1000-1004. doi: 10.1645/GE-1670.1

Jiménez, O. M., Carbonell, A. E., Garcia, O. M., Rodriguez, L. W., Triana, F. P., and Fabián, L. G. (2012). [Blastocystis hominis in symptomatic celiac patients]. Acta Gastroenterol. Latinoam. 42, 175-181.

Kohli, A., Bushen, O. Y., Pinkerton, R. C., Houpt, E., Newman, R. D., Sears, C. L., et al. (2008). Giardia duodenalis assemblage, clinical presentation and markers of intestinal inflammation in Brazilian children. Trans. R. Soc. Trop. Med. Hyg. 102, 718-725. doi: 10.1016/j.trstmh.2008.03.002

Kotloff, K. L., Nataro, J. P., Blackwelder, W. C., Nasrin, D., Farag, T. H., Panchalingam, S., et al. (2013). Burden and aetiology of diarrheal disease in infants and young children in developing countries (the Global Enteric Multicenter Study, GEMS): a prospective, case-control study. Lancet 382, 209-222. doi: 10.1016/S0140-6736(13)60844-2

Leav, B. A., Mackay, M. R., Anyanwu, A., Rm, O. C., Cevallos, A. M., Kindra, G., et al. (2002). Analysis of sequence diversity at the highly polymorphic Cpgp40/15 locus among Cryptosporidium isolates from human immunodeficiency virus-infected children in South Africa. Infect. Immun. 70, 3881-3890. doi: 10.1128/IAI.70.7.3881-3890.2002

Lebbad, M., Ankarklev, J., Tellez, A., Leiva, B., Andersson, J. O., and Svard, S. (2008). Dominance of Giardia assemblage B in Leon, Nicaragua. Acta Trop. 106, 44-53. doi: 10.1016/j.actatropica.2008.01.004

Liu, H., Shen, Y., Yin, J., Yuan, Z., Jiang, Y., Xu, Y., et al. (2014). Prevalence and genetic characterization of Cryptosporidium, Enterocytozoon, Giardia and Cyclospora in diarrheal outpatients in China. BMC Infect. Dis. 14:25. doi: 10.1186/1471-2334-14-25

Malheiros, A. F., Stensvold, C. R., Clark, C. G., Braga, G. B., and Shaw, J. J. (2011). Short report: molecular characterization of Blastocystis obtained from members of the indigenous Tapirape ethnic group from the Brazilian Amazon region, Brazil. Am. J. Trop. Med. Hyg. 85, 1050-1053. doi: 10.4269/ajtmh.2011.11-0481

Mayrhofer, G., Andrews, R. H., Ey, P. L., and Chilton, N. B. (1995). Division of Giardia isolates from humans into two genetically distinct assemblages by electrophoretic analysis of enzymes encoded at 27 loci and comparison with Giardia muris. Parasitology 111 (Pt 1), 11-17. doi: 10.1017/S0031182000064556

Mejia, R., Vicuna, Y., Broncano, N., Sandoval, C., Vaca, M., Chico, M., et al. (2013). A novel, multi-parallel, real-time polymerase chain reaction approach for eight gastrointestinal parasites provides improved diagnostic capabilities to resource-limited at-risk populations. Am. J. Trop. Med. Hyg. 88, 1041-1047. doi: 10.4269/ajtmh.12-0726
Ministerio de Salud y Protección Social (2015). U.D.a.E.N.D.P.I.E.P.E.M.U.D.A. El Ministerio. Available online at: https://www.minsalud.gov.co/sites/rid/Lists/ BibliotecaDigital/RIDE/VS/PP/ET/encuesta-nacional-de-parasitismo-20122014.pdf

Minvielle, M. C., Molina, N. B., Polverino, D., and Basualdo, J. A. (2008), First genotyping of Giardia lamblia from human and animal feces in Argentina, South America. Mem. Inst. Oswaldo Cruz 103, 98-103. doi: 10.1590/S0074-02762008000100015

Monis, P. T., Caccio, S. M., and Thompson, R. C. (2009). Variation in Giardia: towards a taxonomic revision of the genus. Trends Parasitol. 25, 93-100. doi: 10.1016/j.pt.2008.11.006

Montresor, A. (2002). Helminth Control in School-Age Children: a Guide for Managers of Control Programmes. Geneva: World Health Organization.

Nemes, Z. (2009). [Diarrhea from the infectologist's point of view]. Orv. Hetil. 150, 353-361. doi: 10.1556/OH.2009.28549

World Health Organization. (2012). Research Priorities for Helminth Infections: Technical Report of the TDR Disease Reference Group on Helminth Infections. Technical report series; no. 972 II, 196.

Ortiz, C., Lopez, M. C., and Rivas, F. A. (2012). [Helminth prevalence in a waste-water plant at El Rosal, Cundinamarca]. Rev. Salud Publica (Bogota). 14, 296-304. doi: 10.1590/S0124-00642012000200010

Ramírez, J. D., Duque, M. C., Montilla, M., Cucunuba, Z., and Guhl, F. (2012). Natural and emergent Trypanosoma cruzi I genotypes revealed by mitochondrial (Cytb) and nuclear (SSU rDNA) genetic markers. Exp. Parasitol. 132, 487-494. doi: 10.1016/j.exppara.2012.09.017

Ramírez, J. D., Guhl, F., Messenger, L. A., Lewis, M. D., Montilla, M., Cucunuba, Z., et al. (2012). Contemporary cryptic sexuality in Trypanosoma cruzi. Mol. Ecol. 21, 4216-4226. doi: 10.1111/j.1365-294X.2012.05699.x

Ramírez, J. D., Heredia, R. D., Hernandez, C., Leon, C. M., Moncada, L. I. Reyes, P., et al. (2015). Molecular diagnosis and genotype analysis of Giardia duodenalis in asymptomatic children from a rural area in central Colombia. Infect. Genet. Evol. 32, 208-213. doi: 10.1016/j.meegid.2015.03.015

Ramírez, J. D., Hernandez, C., Leon, C. M., Ayala, M. S., Florez, C., and Gonzalez, C. (2016). Taxonomy, diversity, temporal and geographical distribution of Cutaneous Leishmaniasis in Colombia: a retrospective study. Sci. Rep. 6:28266. doi: $10.1038 /$ srep 28266

Ramírez, J. D., Sánchez, A., Hernández, C., Flórez, C., Bernal, M. C., Giraldo, J. C., et al. (2016). Geographic distribution of human Blastocystis subtypes in South America. Infect. Genet. Evol. 41, 32-35. doi: 10.1016/j.meegid.2016. 03.017

Ramírez, J. D., Sanchez, L. V., Bautista, D. C., Corredor, A. F., Florez, A. C., and Stensvold, C. R. (2014). Blastocystis subtypes detected in humans and animals from Colombia. Infect. Genet. Evol. 22, 223-228. doi: 10.1016/j.meegid.2013.07.020

Rinne, S., Rodas, E. J., Galer-Unti, R., Glickman, N., and Glickman, L. T. (2005). Prevalence and risk factors for protozoan and nematode infections among children in an Ecuadorian highland community. Trans. R. Soc. Trop. Med. Hyg. 99, 585-592. doi: 10.1016/j.trstmh.2005.01.003

Roberts, T., Stark, D., Harkness, J., and Ellis, J. (2014). Update on the pathogenic potential and treatment options for Blastocystis sp. Gut. Pathog. 6:17. doi: 10.1186/1757-4749-6-17

Rodríguez, V., Espinosa, O., Carranza, J. C., Duque, S., Arevalo, A., Clavijo, J. A., et al. (2014). [Giardia duodenalis genotypes found in the Instituto Colombiano de Bienestar Familiar day care centers and dogs in Ibague, Colombia]. Biomedica 34, 271-281. doi: 10.7705/biomedica.v34 i2.1713

Rodríguez-Morales, A. J., Granados-Alvarez, S., Escudero-Quintero, H., VeraPolania, F., Mondragon-Cardona, A., Diaz-Quijano, F. A., et al. (2016). Estimating and mapping the incidence of giardiasis in Colombia, 2009-2013. Int. J. Infect. Dis. 49, 204-209. doi: 10.1016/j.ijid.2016.06.005

Ryan, U., and Caccio, S. M. (2013). Zoonotic potential of Giardia. Int. J. Parasitol. 43, 943-956. doi: 10.1016/j.ijpara.2013.06.001

Ryan, U., and Hijjawi, N. (2015). New developments in Cryptosporidium research. Int. J. Parasitol. 45, 367-373. doi: 10.1016/j.ijpara.2015.01.009

Scanlan, P. D., Stensvold, C. R., Rajilic-Stojanovic, M., Heilig, H. G., De Vos, W. M., O'toole, P. W., et al. (2014). The microbial eukaryote Blastocystis is a prevalent and diverse member of the healthy human gut microbiota. FEMS Microbiol. Ecol. 90, 326-330. doi: 10.1111/1574-6941.12396 
Scicluna, S. M., Tawari, B., and Clark, C. G. (2006). DNA barcoding of Blastocystis. Protist 157, 77-85. doi: 10.1016/j.protis.2005.12.001

Sharma, P., Sharma, A., Sehgal, R., Malla, N., and Khurana, S. (2013). Genetic diversity of Cryptosporidium isolates from patients in North India. Int. J. Infect. Dis. 17, e601-e605. doi: 10.1016/j.ijid.2012.12.003

Shirley, D.-A.T., Moonah, S. N., and Kotloff, K.L. (2012). Burden of disease from cryptosporidiosis. Curr. Opin. Infect. Dis. 25:555. doi: 10.1097/QCO.0b013e328357e569

Stensvold, C. R. (2013). Blastocystis: genetic diversity and molecular methods for diagnosis and epidemiology. Trop. Parasitol. 3, 26-34. doi: 10.4103/2229-5070.113896

Stensvold, C. R., Alfellani, M., and Clark, C. G. (2012). Levels of genetic diversity vary dramatically between Blastocystis subtypes. Infect. Genet. Evol. 12, 263-273. doi: 10.1016/j.meegid.2011.11.002

Stensvold, C. R., and Clark, C. G. (2016). Current status of Blastocystis: a personal view. Parasitol. Int. 65, 763-771. doi: 10.1016/j.parint.2016. 05.015

Stensvold, C. R., Elwin, K., Winiecka-Krusnell, J., Chalmers, R. M., Xiao, L., and Lebbad, M. (2015). Development and application of a gp60-Based Typing Assay for Cryptosporidium viatorum. J. Clin. Microbiol. 53, 1891-1897. doi: 10.1128/JCM.00313-15

Stensvold, C. R., Suresh, G. K., Tan, K. S., Thompson, R. C., Traub, R. J., Viscogliosi, E., et al. (2007). Terminology for Blastocystis subtypes-a consensus. Trends Parasitol. 23, 93-96. doi: 10.1016/j.pt.2007.01.004

Tamura, K., Peterson, D., Peterson, N., Stecher, G., Nei, M., and Kumar, S. (2011). MEGA5: molecular evolutionary genetics analysis using maximum likelihood, evolutionary distance, and maximum parsimony methods. Mol. Biol. Evol. 28, 2731-2739. doi: 10.1093/molbev/msr121

Thompson, R. C. (2000). Giardiasis as a re-emerging infectious disease and its zoonotic potential. Int. J. Parasitol. 30, 1259-1267. doi: 10.1016/S0020-7519(00)00127-2

Volotão, A. C., Costa-Macedo, L. M., Haddad, F. S., Brandao, A., Peralta, J. M., and Fernandes, O. (2007). Genotyping of Giardia duodenalis from human and animal samples from Brazil using beta-giardin gene: a phylogenetic analysis. Acta Trop. 102, 10-19. doi: 10.1016/j.actatropica.2007. 02.010

Wang, W., Owen, H., Traub, R. J., Cuttell, L., Inpankaew, T., and BielefeldtOhmann, H. (2014). Molecular epidemiology of Blastocystis in pigs and their in-contact humans in Southeast Queensland, Australia, and Cambodia. Vet. Parasitol. 203, 264-269. doi: 10.1016/j.vetpar.2014.04.006

Wanyiri, J. W., O'connor, R., Allison, G., Kim, K., Kane, A., Qiu, J., et al. (2007). Proteolytic processing of the Cryptosporidium glycoprotein gp 40/15 by human furin and by a parasite-derived furin-like protease activity. Infect. Immun. 75, 184-192. doi: 10.1128/IAI.00944-06

Xiao, L. (2010). Molecular epidemiology of cryptosporidiosis: an update. Exp. Parasitol. 124, 80-89. doi: 10.1016/j.exppara.2009.03.018

Yakoob, J., Jafri, W., Beg, M. A., Abbas, Z., Naz, S., Islam, M., et al. (2010). Blastocystis hominis and Dientamoeba fragilis in patients fulfilling irritable bowel syndrome criteria. Parasitol. Res. 107, 679-684. doi: 10.1007/s00436-010-1918-7

Yan, Y., Su, S., Ye, J., Lai, X., Lai, R., Liao, H., et al. (2007). Blastocystis sp. subtype 5: a possibly zoonotic genotype. Parasitol. Res. 101, 1527-1532. doi: 10.1007/s00436-007-0672-y

Yoder, J. S., Wallace, R. M., Collier, S. A., Beach, M. J., and Hlavsa, M. C. (2012). Cryptosporidiosis surveillance-United States, 2009-2010. MMWR. Surveill. Summ. 61, 1-12.

Conflict of Interest Statement: The authors declare that the research was conducted in the absence of any commercial or financial relationships that could be construed as a potential conflict of interest.

Copyright $\odot 2017$ Sánchez, Munoz, Gómez, Tabares, Segura, Salazar, Restrepo, Ruiz, Reyes, Qian, Xiao, López and Ramírez. This is an open-access article distributed under the terms of the Creative Commons Attribution License (CC BY). The use, distribution or reproduction in other forums is permitted, provided the original author(s) or licensor are credited and that the original publication in this journal is cited, in accordance with accepted academic practice. No use, distribution or reproduction is permitted which does not comply with these terms. 